\title{
Lesões intra-epiteliais vulvares em pacientes infectadas pelo HIV
}

\author{
Vulval intraepithelial lesions in HIV-infected patients
}

\begin{abstract}
Ricardo José de Oliveira e Silva ${ }^{1}$, Maria José Penna Maisonnette de Athayde $^{2}$, Michele Lopes Pedrosa ${ }^{3}$, Susana Cristina Aidé Viviani Fialho ${ }^{4}$, Fabio Bastos Russomano ${ }^{5}$, Beatriz Grinsztejn ${ }^{6}$, Luiz Guilherme Pessoa da Silva ${ }^{7}$
\end{abstract}

\section{RESUMO}

Objetivos: avaliar a prevalência de lesões escamosas intra-epiteliais vulvares em pacientes infectadas pelo HIV atendidas em rede pública na cidade do Rio de Janeiro e estudar os fatores associados a essas lesões. Método: 374 pacientes infectadas pelo HIV e atendidas em serviços públicos na cidade do Rio de Janeiro foram submetidas a exame ginecológico, colheita de citologia e exame colposcópico do colo uterino e vulva. A associação do diagnóstico de HIV com lesão intraepitelial da vulva foi analisada de acordo com de variáveis clínicas (idade e presença de lesões cervicais), laboratoriais (contagem de CD4) e comportamentais (número de parceiros e hábito de fumar). Consideraram-se como variáveis de estudo (independente) os dados epidemiológicos, o status imunológico e o resultado da propedêutica ginecológica. Assim foram selecionados: idade, hábito de fumar, número de parceiros, contagem de linfócitos T CD4 e lesão intra-epitelial cervical. Uma análise bivariada foi inicialmente efetuada, objetivando avaliar a associação entre a presença de lesões intra-epiteliais vulvares (variável de desfecho) e as variáveis independentes (idade, tabagismo, número de parceiros, citologia, colposcopia e contagem de CD4). Em seguida, os resultados de significância estatística $(\mathrm{p} \leq 0,05)$ foram submetidos à regressão logística múltipla, estabelecendo-se as razões de chances com os respectivos intervalos de confiança a 95\%. Resultados: a prevalência de lesões intra-epiteliais vulvares foi de 40\%. Na análise multivariada mostraram-se significativas: contagem de CD4 abaixo de 500 cels $/ \mathrm{mm}^{3}$, OR=2,69 [IC 95\%: 1,61-4,52]; colposcopia anormal, OR=1,64 [IC 95\%: 1,01-2,67] e idade abaixo de 26 anos, $\mathrm{OR}=1,98$ [IC 95\%: 1,18-3,30]. Na análise do subgrupo de pacientes que apresentaram lesões simultâneas no colo e na vulva, mostraram-se significativas no modelo final apenas a idade abaixo de 26 anos, OR=3,30 [IC 95\%: 1,65-6,59], e contagem de CD4 abaixo de 500 cels $/ \mathrm{mm}^{3}, \mathrm{OR}=4,15$ [IC 95\%: 1,92-8,96]. Conclusões: é alta a prevalência de lesões intra-epiteliais vulvares em pacientes infectadas pelo HIV. A imunodeficiência, a presença de lesões intra-epiteliais no colo e a idade inferior a de 26 anos estão associadas à presença de lesões intra-epiteliais da vulva.

PALAVRAS-CHAVE: HIV; Neoplasias vulvares; Colo uterino/patologia

\section{ABSTRACT}

Purpose: to evaluate the prevalence of vulval squamous intraepithelial lesions and associated factors in HIV-infected patients attended at the public health services of Rio de Janeiro city. Method: a total of 374 HIV-infected patients were attended at public services in Rio de Janeiro city and submitted to gynecological examination, Pap smear and colposcopic examination of the cervix and vulva. The association of vulval intraepithelial lesion was analyzed according to the results of clinical (age and cervical lesions), laboratorial (CD4 count) and behavioral (number of partners and smoking habit) variables. The study (independent) variables were the epidemiological data, the immunologic status and the results of gynecological propaedeutic. Thus, age, the smoking habit, number of sexual partners, count of T CD4 lymphocites, and cervical intraepithelial lesion were selected. In the beginning, a bivariate analysis was performed, aiming at assessing the association between the presence of vulval intraepithelial lesion (ultimate variable) and the independent variables (age, smoking habits, number of sexual partners, cytology, colposcopy and CD4 count). Thereafter, the results with statistical significance ( $\mathrm{p} \leq 0.05)$ were submitted to a multiple logistic regression, and the probability ratio with the respective $95 \%$ confidence interval was

Instituto Fernandes Figueira, Hospital Escola São Francisco de Assis e PRO-MATRE

1 Diretor Hospital PRO MATRE - Rio de Janeiro (RJ) - Brasil.

2 Chefe do Setor de Patologia Cervical - Hospital PRO MATRE - Rio de Janeiro (RJ) - Brasil.

3 Médica do Setor de Patologia Cervical - Hospital PRO MATRE - Rio de Janeiro (RJ) - Brasil.

4 Colaboradora em Pesquisa do Setor de Patologia Cervical - Hospital PRO MATRE - Rio de Janeiro (RJ) - Brasil.

5 Chefe do Setor de Patologia Cervical - Instituto Fernandes Figueira, Fundação Oswaldo Cruz - FIOCRUZ - Rio de Janeiro (RJ) - Brasil.

6 Pesquisadora do Instituto Evandro Chagas, Fundação Oswaldo Cruz - FIOCRUZ - Rio de Janeiro (RJ) - Brasil.

7 Coordenador do Centro de Estudos e Pesquisa - Hospital PRO MATRE - Rio de Janeiro (RJ) - Brasil.

Correspondência: Ricardo José de Oliveira e Silva

Associação PRO MATRE

Av. Venezuela, 153, Praça Mauá - 20081-310 - Rio de Janeiro-RJ - Telefone: (21) 2187-1909 - Fax: (21) 2263-1538 - e-mail: rjos@bol.com.br Recebido em: 21/3/2005 
established. Results: the prevalence of vulval intraepithelial lesions was $40 \%$. In the multivariate analysis CD4 count below 500 cells $/ \mathrm{mm}^{3} \mathrm{OR}=2.69$ [IC 95\%: 1.61-4.52], abnormal colposcopy OR=1.64 [IC 95\%: 1.01-2.67] and age under $26 \mathrm{OR}=1.98$ [IC 95\%: 1.18-3.30] were significant. In the vulval and cervical simultaneous lesion subgroup, age under $26 \mathrm{OR}=3.30$ [IC 95\%: 1.65-6.59] and $\mathrm{CD} 4$ count below 500 cells $/ \mathrm{mm}^{3} \mathrm{OR}=4.15$ [IC 95\%: 1.92-8.96], were significant on analysis. Conclusions: the prevalence of vulval squamous intraepithelial lesions in HIV-infected patients is high. Immunodeficiency, presence of cervical intraepithelial lesions and age under 26 were associated with the presence of vulval intraepithelial lesions.

KEYWORDS: HIV; Vulvar neoplasms; Cervix utery/pathology

Introdução

Embora ocorram divergências, a prevalência de lesões induzidas pelo HPV e/ou neoplasia intra-epitelial vulvar (VIN) guarda associação com a imunodeficiência. A prevalência de VIN em pacientes infectadas pelo HIV é difícil de ser estimada, variando entre 5,6 e $37 \%$, de acordo com os critérios adotados ${ }^{1}$. Em pacientes imunodeficientes o risco para o desenvolvimento do câncer de vulva é maior que para o câncer do colo uterino ${ }^{2}$. Nas submetidas a transplante renal o risco é cem vezes maior que na população geral ${ }^{3}$. Da mesma forma que o referido em relação às lesões cérvicouterinas $^{4,5}$, existe a preocupação com o possivel aumento de incidência do câncer de vulva.

Em estudo de seguimento de mulheres infectadas pelo HIV ou imunossuprimidas por medicação foi observada a incidência de $7 \%$ de lesões vulvares incluindo-se dois carcinomas invasores e sete VIN III ${ }^{6}$. A prevalência de lesões intraepiteliais vulvares em pacientes com HIV é bem mais alta que nas não infectadas, estimando-se para as primeiras, taxas sete vezes mais altas. Além disto, cerca de um terço das pacientes com lesões vulvares apresentam lesões cérvicouterinas ${ }^{7}$.

A falta de programas organizados de rastreamento de lesões vulvares faz com que seja dificil estimar a prevalência de lesões intra-epiteliais. A impressão dos autores é de que houve crescimento no número de lesões intra-epiteliais, não havendo correspondente aumento dos casos de carcinoma invasor, talvez pelo pouco tempo decorrido até então ${ }^{8}$.

Dos cerca dos 200.000 casos de AIDS notificados ao Ministério da Saúde de 1980 a dezembro de 2000, 25\% ocorreram em mulheres. De acordo com dados relativos ao município do Rio de Janeiro, cerca de 400 novos casos da infecção por HIV em mulheres serão notificados a cada ano.

A falta de informações sobre a presença de lesões vulvares neste grupo de mulheres levou a desenvolver este estudo. O objetivo foi avaliar a prevalência de lesões escamosas intra-epiteliais vulvares em pacientes infectadas pelo HIV e os fatores de risco associados a essas lesões.

\section{Métodos}

Desenvolvemos estudo de caráter transversal, com investigação observacional em 451 pacientes infectadas pelo HIV, atendidas em serviços públicos de saúde do Rio de Janeiro, no período compreendido entre maio de 1996 e setembro de 2003. As instituições envolvidas foram: Instituto Fernandes Figueira (67\% dos casos), Hospital Escola São Francisco de Assis (29\%) e PRO MATRE (4\%). Em sua origem os casos foram estudados, com outros objetivos, constituindo trabalhos já publicados $^{9,10}$.

A população, extraída de coortes de pacientes infectadas pelo HIV oriundas das instituições envolvidas, foi submetida a estudo descritivo e analítico. A primeira parte do estudo compreendeu enfoque descritivo, determinando-se a prevalência do desfecho (lesão intra-epitelial de vulva) e das características clínicas, laboratoriais e comportamentais. As variáveis clínicas foram obtidas por meio do exame físico, ginecológico e colposcópico de todas as pacientes. As variáveis ditas comportamentais foram obtidas por meio de questionários. O tabagismo foi considerado de maneira dicotômica pela condição atual de fumante, não sendo aferida a quantidade consumida. O número de parceiros foi considerado pela informação de toda a vida sexual referida. Na segunda parte, valendo-nos de estudo analítico, avaliamos a associação entre o desfecho e um grupo de variáveis selecionadas da literatura pertinente e disponíveis para análise.

Foram incluidas mulheres infectadas (independentemente do estágio clínico e do tipo de tratamento), de qualquer idade, que freqüentavam regularmente o programa especializado para 
acompanhamento de portadoras do HIV. Foram excluídas aquelas cujos prontuários apresentaram informações imprecisas ou dados incompletos. Do consolidado dos bancos de dados de mulheres infectadas pelo HIV, assistidas em serviços públicos do Rio de Janeiro, foram identificadas 451 pacientes, das quais 374 formaram o grupo de estudo.

Para o estabelecimento do diagnóstico da infecção causada pelo HIV foram considerados dois testes positivos pelo método ELISA e um teste confirmatório pela técnica de imunofluorescência indireta ou Western-blot, conforme normas estabelecidas pelo programa de AIDS do Ministério da Saúde do Brasil ${ }^{11}$. O status imunológico foi caracterizado pela contagem de linfócitos CD4, pela técnica Becton Dickinson F ACScan, San Jose, CA, USA, valorizando-se os dados laboratoriais contemporâneos aos resultados da citologia, da colposcopia e da biópsia.

Os exames colposcópicos foram realizados em todas as pacientes e seguiram a rotina tradicional para o procedimento, incluindo-se exame obrigatório do colo e da vulva. Utilizou-se, inicialmente, o soro fisiológico a 0,9\%, em seguida, o ácido acético a 5\% e, por fim, a solução de Lugol para realizar o teste de Schiller. Na vulva, região perineal e perianal, o exame foi realizado utilizando-se, somente, solução de ácido acético a $5 \%$. As biópsias cervicais ou vulvares foram indicadas quando a colposcopia revelava imagem sugestiva de lesão intra-epitelial. O procedimento foi efetuado com troca de Baliú ou alça diatérmica e os espécimes eram colocados em formol tamponado e corados pelo método de hematoxilina-eosina. As análises foram feitas nos laboratórios de anatomia patológica das instituições envolvidas.

Consideraram-se como variáveis de estudo (independente) os dados epidemiológicos, o status imunológico e o resultado da propedêutica ginecológica. Assim, foram selecionados: idade, hábito de fumar, número de parceiros, contagem de linfócitos T CD4 e lesão intra-epitelial cervical.

Consideramos ausência de lesão intraepitelial do colo quando a colposcopia era satisfatória (junção escamo-colunar visível) e não eram observadas atipias epiteliais, ou quando, apesar de serem observadas imagens anormais, o diagnóstico histológico da biópsia não revelava lesão intra-epitelial. Foi considerada positiva, quando a colposcopia ou algum espécime histológico evidenciava lesão intra-epitelial, incluindo-se as lesões compatíveis com a infecção pelo HPV.
Considerou-se como desfecho (variável dependente) a ocorrência da lesão intra-epitelial escamosa da vulva, considerada presente (positivo) quando observadas lesões sugestivas de alteração neoplásica ou compativeis com infecção pelo HPV, seja pela vulvoscopia ou pela análise de espécime obtido por biópsia.

Uma análise bivariada foi inicialmente efetuada, objetivando avaliar a associação entre a presença de lesões intra-epiteliais vulvares (variável de desfecho) e as variáveis independentes (idade, tabagismo, número de parceiros, citologia, colposcopia e contagem de CD4). Em seguida, os resultados de significância estatística $(p \leq 0,05)$ foram submetidos à regressão logística múltipla, estabelecendo-se as razões de chances com os respectivos intervalos de confiança a 95\%.

Para a análise estatística utilizou-se o aplicativo Statistical Package for Social Science (SPSS), versão 8.0 para Windows.

\section{Resultados}

No que tange às características epidemiológicas, observou-se que a idade da população estudada variou de 16 a 67 anos, tendo como média 33,9 ( $\pm 10,2$ anos). A mediana e a moda foram de 33 anos. Observou-se comportamento normal da distribuição da idade das pacientes, aglutinando maior número de casos em torno da média, mediana e moda. Subordinando essa população à estratificação por categoria, em ponto de corte próximo da média (33 anos), obteve-se prevalência de $48,5 \%$ dos casos abaixo da referência.

A variável "hábito de fumar" esteve presente em 40,3\% das pacientes. Observou-se ainda que $62 \%$ da população revelaram ter tido mais de três parceiros sexuais durante a vida e $32,2 \%$ afirmaram ter se relacionado sexualmente com mais de sete parceiros (pontos de corte arbitrados em outros estudos) ${ }^{10,11}$. Observou-se que as prevalências das alterações citológicas e colposcópicas na população soropositiva foram, respectivamente, $40 \mathrm{e}$ $38,8 \%$. Considerando-se os pontos de corte da contagem do CD4 em 500, 350 e 200 células por mm³, observou-se que, respectivamente, $67,8,49,2$ e $25,0 \%$ da população estavam com valores considerados anormais por estarem abaixo desses pontos de corte (Tabela 1). Observou-se que $35,8 \%$ da população apresentavam lesão epitelial do colo uterino. 
Tabela 1 - Distribuição da freqüência das variáveis clínicas, laboratoriais e comportamentais em pacientes portadoras do HIV atendidas em serviços públicos da cidade do Rio de Janeiro (análise de 374 casos).

\begin{tabular}{|c|c|c|}
\hline Situação & $\begin{array}{c}\text { Freqüência } \\
\text { absoluta }\end{array}$ & $\begin{array}{c}\text { Freqüência } \\
\text { relativa }\end{array}$ \\
\hline Idade $<33$ anos & 181 & 48,5 \\
\hline Tabagismo & 140 & 40,3 \\
\hline $\mathrm{N}^{0}$ parceiros $>3$ & 227 & 62,0 \\
\hline $\mathrm{N}^{0}$ parceiros $>7$ & 118 & 32,3 \\
\hline Citologia anormal & 148 & 40,0 \\
\hline Colposcopia anormal & 144 & 38,8 \\
\hline Contagem de CD4 <500/mm³ & 250 & 67,8 \\
\hline Contagem de $\mathrm{CD} 4<350 / \mathrm{mm}^{3}$ & 182 & 49,2 \\
\hline Contagem de CD4 <200/mm & 92 & 25,0 \\
\hline Lesão epitelial do colo uterino & 134 & 35,8 \\
\hline
\end{tabular}

A prevalência da lesão intra-epitelial de vulva na população portadora do HIV, atendida em serviços públicos do Rio de Janeiro, foi de 40,0\% (IC 95\%: 31,5-48,4).

Com o objetivo de avaliar o poder de associação entre os fatores de natureza epidemiológica, imunológica e da propedêutica genital nas pacientes portadoras do HIV e o desfecho lesão intraepitelial de vulva, estabeleceram-se análises bivariadas, tendo de um lado, como variáveis independentes, aquelas descritas no capítulo Métodos e, como dependente, a lesão intra-epitelial de vulva, dicotomizada em presente e ausente.

A distribuição das análises bivariadas mostra que a variável contínua idade, quando transformada em discreta, por meio de análises sucessivas a partir da média, revelou haver associação significativa com o desfecho anunciado, somente na faixa etária inferior a 26 anos $(p=0,0043)$. O número de parceiros sexuais, utilizando pontos de corte de três $(p=0,9605)$ e de sete parceiros $(p=0,7076)$, não revelou associação com o desfecho. O mesmo ocorreu com a variável hábito de fumar, que não mostrou associação com o aparecimento da lesão intra-epitelial de vulva em mulheres portadoras do HIV ( $p=0,9536)$. Ficou demonstrado ainda que os resultados citológicos $(p=0,0098)$ e colposcópicos anormais ( $\mathrm{p}=0,0014)$ estavam significativamente associados à presença de lesão vulvar. A contagem de CD4 revelou associação significativa com a lesão intra-epitelial da vulva, quando foram considerados como valores anormais os pontos de corte abaixo de $350(\mathrm{p}=0,0100)$ e de 500 cels $/ \mathrm{mm}^{3}$ de sangue $(\mathrm{p}=0,0000)$. O mesmo não aconteceu quando o CD4 era inferior a 200 cels/ $\mathrm{mm}^{3}$ de sangue $(\mathrm{p}=0,3297)$, talvez explicado pelo pequeno número de casos (Tabela 2 ).

Tabela 2 - Distribuição das variáveis na população estudada, segundo análise bivariada, tendo como desfecho a ocorrência de lesão intra-epitelial de vulva em portadoras do HIV.

\begin{tabular}{|c|c|c|c|c|c|c|}
\hline \multirow[t]{2}{*}{ Variáveis } & \multicolumn{2}{|c|}{ Lesão intra-epitelial de vulva } & \multirow[t]{2}{*}{ Total } & \multirow[t]{2}{*}{ Razão de chances } & \multirow{2}{*}{$\begin{array}{l}\text { Razão de chances } \\
\text { (IC 95\%) }\end{array}$} & \multirow[t]{2}{*}{$\mathrm{p}$} \\
\hline & Sim & Não & & & & \\
\hline Idade $<33$ anos & $78 / 180$ & $70 / 189$ & 369 & 1,30 & $0,83-2,03$ & 0,2596 \\
\hline Idade $<26$ anos & $49 / 92$ & $99 / 277$ & 369 & 2,05 & $1,23-3,41$ & 0,0043 \\
\hline Tabagismo & $58 / 139$ & $87 / 204$ & 343 & 0,96 & $0,60-1,53$ & 0,9536 \\
\hline$>3$ parceiros & $90 / 224$ & $55 / 138$ & 362 & 1,01 & $0,64-1,61$ & 0,9605 \\
\hline$>7$ parceiros & $49 / 117$ & $96 / 245$ & 362 & 1,12 & $0,69-1,80$ & 0,7076 \\
\hline Citologia anormal & $79 / 166$ & $69 / 204$ & 370 & 1,78 & $1,14-2,78$ & 0,0098 \\
\hline Colposcopia anormal & $77 / 155$ & $67 / 206$ & 361 & 2,05 & $1,30-3,23$ & 0,0014 \\
\hline CD4 $<500$ & $118 / 248$ & $30 / 119$ & 367 & 2,69 & $1,61-4,52$ & 0,0000 \\
\hline CD4 $<350$ & $85 / 180$ & $63 / 188$ & 368 & 1,78 & $1,14-2,78$ & 0,0100 \\
\hline CD4 $<200$ & $41 / 91$ & $106 / 275$ & 366 & 1,31 & $0,78-2,18$ & 0,3297 \\
\hline
\end{tabular}

$\mathrm{p} \leq 0,05=$ significativo.

Com o objetivo de avaliar, em conjunto, o poder de associação das variáveis analisadas, os dados foram submetidos a ajustamento multivariável, passo a passo, modulando as variáveis independentes que mostraram associação significativa com o desfecho (Tabela 3). Realizaram-se várias simulações no modelo, visando a escolha do mais representativo, sob o ponto de vista da explicação.
Tabela 3 - Distribuição das variáveis na população estudada, segundo análise multivariada (modelo final), tendo como desfecho a ocorrência de lesão intra-epitelial de vulva em portadoras do HIV (taxa de acerto de 67,49\%).

\begin{tabular}{|c|c|c|c|c|}
\hline \multirow[t]{2}{*}{ Variável } & $\beta$ & Razão & Razão & $p$ \\
\hline & \multicolumn{4}{|c|}{$\begin{array}{l}\text { de chances de chances } \\
\text { (IC 95\%) }\end{array}$} \\
\hline Idade $<26$ anos & 0,6848 & 1,98 & $1,18-3,30$ & 0.0087 \\
\hline Colposcopia anormal & 0,4975 & 1,64 & $1,01-2,67$ & 0,0452 \\
\hline CD4 $<500$ & 1,1706 & 3,22 & $1,92-5,40$ & 0,0000 \\
\hline
\end{tabular}

$p \leq 0,05=$ significativo. 
No modelo final o aparecimento da lesão epitelial da vulva foi influenciada pelas seguintes variáveis: idade menor que 26 anos, CD4 inferior a 500 cels $/ \mathrm{mm}^{3}$ e colposcopia cérvico-uterina anormal. A idade inferior a 26 anos, que revelou associação na análise bivariada, permaneceu no modelo, contribuindo de forma significativa com a presença do desfecho. O achado citológico anormal, quando analisado em conjunto com outras variáveis, perdeu significância estatística, sendo portanto excluído do modelo final. O exame colposcópico anormal permaneceu exibindo associação com a lesão de vulva em todas as fases do processo. A contagem de células CD4, analisadas em vários pontos de corte, revelou que o número de células inferior a 350 influenciou negativamente em outras variáveis. A substituição da variável pelo CD4 $<500$ cels $/ \mathrm{mm}^{3}$ melhorou a precisão do modelo para explicar o desfecho (de 64,99 para $67,49 \%)$.

Dado o interesse pela explicação dos casos mais graves, foi realizada análise de um subgrupo da população de estudo, formada por 236 pacientes soropositivas, comparando as que apresentaram lesões simultâneas no colo e na vulva (73), com aquelas em que não foi diagnosticada qualquer das lesões (163).

Seguindo a mesma metodologia, os dados foram submetidos a análises bivariadas, considerando como dependente a concomitância das referidas lesões e, como independentes, as seguintes variáveis: idade $<26$, idade $<33$ anos, tabagismo, número de parceiros sexuais $>3 \mathrm{e}>7$ e contagem de CD4 $<200$ cels $/ \mathrm{mm}^{3},<350$ cels $/ \mathrm{mm}^{3}$ e $<500 \mathrm{cels} / \mathrm{mm}^{3}$. Uma vez efetivadas as análises ficou demonstrado que as variáveis: idade $<26$ anos e $<33$ anos e nivel de CD4 inferior a 350 e 500 cels $/ \mathrm{mm}^{3}$ apresentaram associação significativa com a presença concomitante de lesão intra-epitelial de vulva e colo. Por outro lado, ficou evidenciado que o número de parceiros sexuais (>3 e >7), o hábito de fumar e a contagem de CD4 inferior a 200 células $/ \mathrm{mm}^{3}$ de sangue não se associaram com o já referido desfecho (Tabela 4).

Tabela 4 - Distribuição das variáveis do subgrupo da população estudada, segundo análise bivariada, tendo como desfecho a ocorrência de lesões simultâneas do colo uterino e da vulva em portadoras do HIV.

\begin{tabular}{lcccccc}
\hline Variáveis & $\begin{array}{c}\text { Lesão simultânea do colo e da vulva } \\
\text { nim }\end{array}$ & Total & Razão de chances & $\begin{array}{c}\text { Razão de chances } \\
\text { (IC 95\%) }\end{array}$ & $p$ \\
\hline Idade $<33$ anos & $45 / 118$ & $28 / 117$ & 235 & 1,96 & $1,07-3,61$ & 0,0269 \\
Idade $<26$ anos & $29 / 58$ & $44 / 133$ & 235 & 3,02 & $1,55-5,91$ & 0,0006 \\
Tabagismo & $37 / 89$ & $36 / 124$ & 213 & 1,74 & $0,94-3,23$ & 0,0791 \\
$\quad>3$ parceiros & $49 / 152$ & $24 / 80$ & 232 & 1,11 & $0,59-2,10$ & 0,8414 \\
$>7$ parceiros & $25 / 81$ & $48 / 151$ & 232 & 0,96 & $0,51-1,80$ & 0,9969 \\
CD4 <500 & $63 / 158$ & $10 / 76$ & 234 & 4,38 & $1,98-9,90$ & 0,0000 \\
CD4 <350 & $50 / 113$ & $23 / 121$ & 234 & 3,38 & $1,80-6,39$ & 0,0000 \\
CD4 $<200$ & $23 / 59$ & $49 / 174$ & 233 & 1,63 & $0,83-3,18$ & 0,1640 \\
\hline
\end{tabular}

$p \leq 0,05=$ significativo.

Nos sucessivos ajustamentos, com vista ao modelo final, foram incluídas todas as variáveis que mostraram significância estatística $(p<0,05)$ na análise bivariada. Além destas, a variável hábito de fumar foi introduzida na análise. A decisão foi estribada no interesse clínico e na proximidade do limite de probabilidade desejado.

O modelo final demonstra associação significativa entre as variáveis: idade inferior a 26 anos $(p=0,0007)$ e contagem de CD4 abaixo de 500 cels / $\mathrm{mm}^{3}(\mathrm{p}=0,0248)$ com a presença de lesões concomitantes da vulva e colo uterino.

\section{Discussão}

A construção do grupo de pacientes em estudo, conforme descrito em capítulo pertinente, resultou de um consolidado de dados colhidos de diferentes serviços dedicados ao acompanhamento de pacientes infectadas pelo HIV na cidade do Rio de Janeiro.

O perfil sociodemográfico da população deste estudo é assemelhado ao da média da população do Rio de Janeiro e municípios periféricos: cerca de $65 \%$ dos indivíduos com até oito anos de freqüência escolar e renda familiar em torno de três salários minimos ${ }^{12}$. Esse aspecto difere dos estudos realizados na França e Estados Unidos. 
Apenas $17,2 \%$ dos casos apontaram o uso de drogas injetáveis como fator de risco para a transmissão do vírus. Com efeito, o contato heterossexual foi a forma predominante de transmissão. Note-se que $38 \%$ das pacientes em estudo relataram menos de três parceiros na vida.

O período de avaliação dos casos incluídos, a nosso juízo, não traz o prejuízo de grandes mudanças relevantes na conduta diagnóstica, intervenções ou perfil da doença. Tanto o método de diagnosticar a infecção pelo HIV, quanto os meios de detecção das lesões do epitélio vulvar e cérvicouterino foram semelhantes, de forma que o grupo pode ser considerado homogêneo sob este aspecto.

Os diagnósticos citológicos e colposcópicos foram reclassificados para permitir agrupamento e análise estatística. Embora tenhamos coletado, originalmente, as denominações oriundas das diversas classificações, o critério para considerar presente a lesão intra-epitelial, englobando as lesões compativeis com a infecção pelo HPV, era de fácil identificação nos laudos.

A prevalência de lesões intra-epiteliais do colo uterino, em nosso material $(35,5 \%)$, situa-se entre as mais elevadas dentre as publicadas. Notese que a prevalência dessas lesões, detectadas pela citologia, no Rio de Janeiro, é de $2 \%{ }^{13}$.

A prevalência de lesões vulvares (40\%) nos parece bastante elevada, embora não existam dados de prevalência da população geral.

Existem poucos estudos que permitam avaliar a prevalência das lesões vulvares em pacientes infectadas pelo HIV ${ }^{1}$. A falta do exame colposcópico, sistemático, da região vulvar dessas mulheres pode explicar as diferentes prevalências relatadas. Nos estudos de prevalência das lesões do colo e da vulva revelam-se alguns problemas comuns: a seqüência temporal, o estágio de evolução da imunodeficiência, o tempo de infecção pelo HIV e os critérios e métodos diagnósticos das lesões intra-epiteliais.

Ao estudarmos a prevalência de uma condição associada à atividade sexual em amostra formada por mulheres que freqüentam clínicas de DST, é esperada uma freqüência aumentada. Da mesma forma, se as lesões epiteliais do trato genital inferior estão associadas à infecção pelo HIV, é provável que a prevalência seja maior em indivíduos infectados há mais tempo. Assumimos, conforme relatado na metodologia, como positivos, ou seja, lesão intra-epitelial presente, aqueles casos em que foram diagnosticadas alterações compativeis com a infecção pelo HPV. Tal decisão encontra respaldo, como exposto em nossa funda- mentação, na tendência atual de incluir essas lesões no grupo das lesões intra-epiteliais escamosas de baixo grau ${ }^{14,15}$ ou VIN I ${ }^{16}$.

A idade, estudada como variável de risco neste trabalho, assume importância maior na medida em que, ao contrário da esperada correlação linear entre o seu aumento e a prevalência das lesões epiteliais, aqui observamos o revés. A idade menor que 33 anos, mediana da amostra, mostrou-se como fator de risco tanto para o desfecho de lesão intra-epitelial cervical quanto vulvar. Mais que isso, a idade abaixo de 26 anos mostrou-se de maior significância. Podemos relacionar nossos resultados à tendência, observada na incidência de lesões ditas precursoras, de aumento da prevalência do HPV, em especial nas pacientes infectadas pelo HIV.

A prevalência do HPV, considerado o principal agente causal do câncer do colo, é mais elevada entre os 20 e 29 anos de idade, o que faz supor que o início precoce da atividade sexual resultaria em maior risco de infecção e desenvolvimento de neoplasias ${ }^{17}$.

Entendemos que o número de parceiros é variável de dificil aferição e que sofre a influência da idade atual, bem como do início da atividade sexual. Ressalte-se que é dificil alguém lembrar o número total de parceiros durante toda a vida, principalmente, se foram muitos. $O$ aspecto cultural pode, também, introduzir outro vício de aferição: os homens tendendo a aumentar e as mulheres tendendo a diminuir o número de parceiros.

Em relação ao tabagismo, presente em 40,3\% das pacientes, optamos pelo critério de aferir o uso atual do tabaco, não considerando o seu uso no passado, nem a quantidade de cigarros. Esta variável não se mostrou significativa na análise univariada $(p=0,9536)$.

Os estudos de prevalência e incidência de lesões epiteliais, em pacientes infectadas pelo HIV, talvez pela grande proporção de fumantes nos grupos de análise, não demonstraram associação estatisticamente significativa. É possivel que estejam refletidos confundimento ou colinearidade devido à presença dos outros fatores na população de fumantes.

O estado imunológico foi analisado mediante contagem dos linfócitos T CD4, células alvo do HIV e principal parâmetro laboratorial de acompanhamento da infecção. Nossa amostra contou com $67,8 \%$ de pacientes com contagem abaixo de 500 cels $/ \mathrm{mm}^{3}, 49,2 \%$ abaixo de 350 cels $/ \mathrm{mm}^{3}$ e $25 \%$ abaixo de 200 cels $/ \mathrm{mm}^{3}$. São considerados valores normais os acima de 500 cels $/ \mathrm{mm}^{3}$. Classica- 
mente, os pontos de corte para a tomada de decisões clínicas ou estadiamento da doença são de 500 e 200 cels $/ \mathrm{mm}^{3}$. Contudo, considera-se hoje, como parâmetro indicativo de início de terapia anti-retroviral, a contagem de linfócitos T CD4 abaixo de 350 cels $/ \mathrm{mm}^{3}$.

Em nosso material, tanto o grupo com menos de 500 cels $/ \mathrm{mm}^{3}$, quanto as com menos de 350 cels $/ \mathrm{mm}^{3}$, demonstraram associação com o desfecho. O grupo com menos de 200 cels $/ \mathrm{mm}^{3}$ não mostrou associação significante, talvez pelo pequeno número de indivíduos com essa contagem. Embora não tenhamos aferido a variável tempo de doença, é possivel que o grupo de mulheres do nosso estudo tenha procurado mais precocemente os serviços de saúde ${ }^{3,4}$.

A presença de lesões simultâneas do colo e da vulva foi detectada em 73 pacientes. No interesse de verificar os possiveis fatores associados a essa ocorrência seguimos a mesma metodologia de análise. Restaram, como variáveis significativas, e com melhor capacidade de explicar esse desfecho no modelo final, a idade abaixo de 26 anos e a contagem de CD4 abaixo de 500 cels $/ \mathrm{mm}^{3}$.

A comparação desse resultado com a literatura é difícil, devido à escassez de estudos publicados a tratar dessa análise, especificamente. Atenção especial deve ser conferida à adesão de pacientes infectadas ao acompanhamento e tratamento. Há indícios de que a terapia anti-retroviral diminui a incidência dessas lesões ${ }^{18}$. As lesões do epitélio vulvar não foram avaliadas, na literatura compulsada, como fator associado às lesões do colo uterino. Entendemos que este é um parâmetro clínico útil, e de fácil aferição pela colposcopia. Por fim, a freqüência aumentada de lesões epiteliais da vulva, em pacientes infectadas pelo HIV, bem como a freqüente simultaneidade com as lesões do colo, tornam obrigatória a sua acurada avaliação. A alta prevalência de lesões epiteliais em mulheres infectadas pelo HIV obriga maior vigilância, propedêutica especializada e exame cuidadoso em todos os níveis do trato genital inferior.

\section{Referências}

1. Spitzer M. Lower genital tract intraepithelial neoplasia in HIV-infected women: guidelines for evaluation and management. Obstet Gynecol Surv. 1999;54(2):131-7.

2. Einstein $\mathrm{MH}$, Kadish AS. Anogenital neoplasia in AIDS. Curr Opin Oncol. 2004;16(5):455-62.

3. Penn I. Tumors of the immunocompromised patient. Ann Rev Med. 1988;39:63-73.

4. Petry KU, Kochel H, Bode U, Schedel I, Niesert S, Glaubitz M, et al. Human papillomavirus is associated with the frequent detection of warty and basaloid high-grade neoplasia of the vulva and cervical neoplasia among immunocompromised women. Gynecol Oncol. 1996;60(1):30-4.

5. Chiasson MA, Ellerbrock TV, Bush TJ, Sun XW, Wright TC Jr. Increased prevalence of vulvovaginal condyloma and vulvar intraepithelial neoplasia in women infected with the human immunodeficiency virus. Obstet Gynecol. 1997;89(5 Pt 1):690-4.

6. Singer A, Monaghan JM. Colposcopia: patologia e tratamento do trato genital inferior. 2a ed. Rio de Janeiro: Revinter; 2002.

7. Fialho SCAV. Prevalência das neoplasias intraepiteliais cervicais e lesões induzidas pelo HPV nas mulheres HIV soro-positivas/AIDS [dissertação]. Rio de Janeiro: Universidade Federal do Rio de Janeiro; 2000.

8. Grinsztejn B. Prevalência de infecções sexualmente transmissiveis e estudo dos fatores de risco para infecção pelo HPV numa coorte de mulheres infectadas pelo HIV no Rio de Janeiro [tese]. Rio de Janeiro: Universidade Federal do Rio de Janeiro; 2001.

9. Ministério da Saúde. Coordenação Nacional de DST/AIDS. Manual de controle das doenças sexualmente transmissíveis. Brasília (DF): Ministério da Saúde; 2000.

10.Six C, Heard I, Bergeron C, Orth G, Poveda JD, Zagury $\mathrm{P}$, et al. Comparative prevalence, incidence and short-term prognosis of cervical squamous intraepithelial lesions amongst HIVpositive and HIV-negative women. AIDS. 1998;12(9): 1047-56.

11.Ellerbrock TV, Chiasson MA, Bush TJ, Sun XW, Sawo D, Brudney K, et al. Incidence of cervical squamous intraepithelial lesions in HIV-infected women. JAMA. 2000;283(8):1031-7. 
12.Instituto Brasileiro de Geografia e Estatística [homepage da Internet]. Pesquisa Nacional por Amostra de Domicílios; 1997 [citado 2005 Jan 20]. Disponivel em: http://www.ibge.gov.br

13. Ministério da Saúde. Instituto Nacional do Câncer. Dados da produção do Serviço Integrado Tecnológico em Citopatologia (SITEC). 1999-2000. Brasília (DF); 2001.

14. Richart RM. A modified terminology for cervical intraepithelial neoplasia. Obstet Gynecol. 1990;75(1):131-3.

15. Solomon D, Davey D, Kurman R, Moriarty A, O'Connor D, Prey M, et al. The 2001 Bethesda System: terminology for reporting results of cervical cytology. JAMA. 2002;287(16):2114-9.
16. International Society for the study for vulvovaginal disease [homepage on the Internet]. New information on vin terminology (2001 ISSVD proposal). 2002 [cited 2005 Jan 20]. Available from: http: / / www.asccp.org/edu/practice/vulva/ hpv-_vin/vin_def_newterm.shtml

17. International Agency for Research on Cancer Working Group on the Evaluation of Carcinogenic Risks to Humans. Human papillomaviruses. Lyon: IARC; 1995. (IARC Monographs on the Evaluation of Carcinogenic Risks to Humans, 65).

18. Massad LS, Silverberg MJ, Springer G, Minkoff H, Hessol N, Palefsky JM, et al. Effect of antiretroviral therapy on the incidence of genital warts and vulvar neoplasia among women with the human immunodeficiency virus. Am J Obstet Gynecol. 2004;190(5):1241-8. 